\title{
How to Measure Reproductive Success?
}

\author{
BEVERLY I. STRASSMANN ${ }^{1 *}$ AND BRENDA GILLESPIE ${ }^{2}$ \\ ${ }^{1}$ Department of Anthropology, University of Michigan, Ann Arbor, Michigan 48109 \\ ${ }^{2}$ Department of Biostatistics, School of Public Health, and Center for Statistical Consultation and \\ Research, University of Michigan, Ann Arbor, Michigan 48109
}

\begin{abstract}
To date there have been few empirical comparisons between alternative methods for measuring reproductive success (RS). We consider the pros and cons of alternative measures of RS to provide guidance for the design of field studies in human behavioral ecology. We compare cross-sectional measures that count offspring alive at the time of the interview and retrospective measures that require data on offspring age at death or censoring. We consider analyses that include adult women (yielding age-specific estimates of RS) as well as analyses restricted to postreproductive women (yielding data on lifetime RS). These methods are applied to reproductive data for the Dogon of Mali, West Africa. Am. J. Hum. Biol. 15:361-369, 2003.
\end{abstract}

Measures of reproductive success (RS) play an important role in human behavioral ecology, but sometimes lack rigor or are subject to hidden biases. This article discusses the strengths and weaknesses of alternative methods for measuring RS. All of these measures are heuristic attempts to understand Darwinian fitness, defined as an individual's genetic contribution to future generations. Behavioral ecologists working with living subjects usually have reasonably complete data on the offspring generation, but not grandoffspring and subsequent generations (especially for younger cohorts). They also usually have data only on direct reproduction (via offspring) and not on indirect reproduction (via aid to nieces, nephews, or other kin). By necessity, therefore, measures of reproductive success attempt to estimate a plausible proxy for fitness, such as the number of offspring who survived to maturity, or age 20 years. In most analyses, reproductive success is an outcome variable and the objective is to identify the predictor variables that are important in a given population during the time of fieldwork.

We consider the pros and cons of alternative measures of $\mathrm{RS}$ to provide guidance both for the design of field studies and for the analysis of reproductive data. We compare cross-sectional measures that count offspring alive at the time of the interview and retrospective measures that require data on age at death or censoring. We consider analyses that include adult women (yielding agespecific estimates of $\mathrm{RS}$ ) as well as analyses restricted to postreproductive women (yielding data on lifetime RS). The relationships between these various measures are shown in Table 1. At the outset, we emphasize that measures of RS may suffer from a great many potential biases not considered here. These other issues include paternity certainty, recall or reporting biases, and birth timing (for example, early reproducers may achieve greater genetic representation in future gene pools). Our present focus is limited to a comparison of the methods in Table 1.

To explore the effectiveness of these methods for identifying predictors of RS, we applied them to reproductive data for the Dogon of Mali, West Africa. Ethnographic information on our study population and the methods of data collection in the field are described elsewhere (Strassmann and Warner, 1998; Strassmann, 1997, 2000; Strassmann and Gillespie, 2002).

\section{LIFETIME RETROSPECTIVE METHOD}

The lifetime retrospective method is the only method that potentially calculates lifetime reproductive success instead of estimating it. The first task for researchers using this method is to define the age to which offspring survival is measured. Common choices are 5 years, 10 years, and 15 years. The older the age, the better the estimate for

Contract grant sponsor: the LSB Leakey Foundation Contract grant numbers: NSF BNS-8612291, NSF SBR9727229

*Correspondence to: B.I. Strassman, Department of Anthropology, University of Michigan, Ann Arbor, MI 48109.

Received 16 August 2002; Accepted 9 December 2002

Published online in Wiley InterScience (www.interscience. wiley.com). DOI: 10.1002/ajhb.10154 
TABLE 1. Relationships between different measures of $R S$

\begin{tabular}{|c|c|c|c|c|}
\hline \multicolumn{3}{|c|}{ Retrospective data } & \multicolumn{2}{|l|}{ Cross-sectional data } \\
\hline Adult women ${ }^{a}$ & $\begin{array}{l}\text { Kaplan Meier-adjusted } \\
\left(\mathrm{n}=104, \mathrm{R}^{2}=0.46\right)\end{array}$ & $\begin{array}{l}\text { Traditional } \\
\left(\mathrm{n}=104, \mathrm{R}^{2}=0.48\right)\end{array}$ & $\begin{array}{l}\text { Age-specific cross-sectional } \\
\left(\mathrm{n}=150, \mathrm{R}^{2}=0.66\right)\end{array}$ & $\begin{array}{l}\text { Age-specific } \\
\text { RS }\end{array}$ \\
\hline $\begin{array}{l}\text { Postreproductive } \\
\text { women }^{\mathrm{b}}\end{array}$ & \multicolumn{2}{|c|}{$\begin{array}{l}\text { Lifetime retrospective } \\
\text { (gold standard) } \\
\left(\mathrm{n}=42, \mathrm{R}^{2}=0.52\right)\end{array}$} & $\begin{array}{l}\text { Lifetime cross-sectional } \\
\left(\mathrm{n}=42, \mathrm{R}^{2}=0.51\right)\end{array}$ & $\begin{array}{l}\text { Lifetime } \\
\text { RS }\end{array}$ \\
\hline
\end{tabular}

${ }^{\mathrm{a}}$ May be limited, for example, to women age 20 years and older or to women whose oldest offspring was born 10 years prior to her last interview.

${ }^{\mathrm{b}}$ Ideally, $\mathrm{x}$ years since the birth of last child if survivorship is measured to age $\mathrm{x}$.

The sample sizes and $\mathrm{R}^{2}$ for the models presented in Tables 3 and 4 are for Dogon data. The retrospective methods have the least risk of bias, but the cross-sectional methods are less onerous and gave similar results. See headings for pertinent sections of this article.

survival to sexual maturity, but the smaller the sample size. The next task is to limit the sample so that it includes only those subjects who have completed reproduction and whose youngest child has had time to reach the age to which survival is measured, regardless of whether he or she survived. Thus, the sample should be restricted to those mothers who last gave birth at least $x$ years prior to the interview, with survival measured to age $x$. Even if the lastborn child died, it is important that at least $x$ years have elapsed since her birth in order for her mother to be included in the sample. Otherwise the sample will be biased toward parents of deceased offspring. For each woman included in the sample, $\mathrm{RS}$ is the number of offspring who survived to age $x$.

The common practice of simply restricting the sample to postmenopausal women may lead to bias if some of the women who have reached menopause have offspring who have not yet reached the age to which survival is measured. Moreover, in most cases fertility ceases well before actual menopause, so restricting the sample to postmenopausal women will lead to an unnecessary reduction in sample size. In a noncontracepting population such as the Dogon, 5 years without a birth usually indicates that a woman is postreproductive (assuming she has a resident spouse). In a contracepting population, it may be preferable to choose a fixed age, such as 45 years, as a proxy for the end of reproduction. (An earlier age will increase sample size and reduce bias caused by the omission of deceased women. A later age will give greater certainty that all births have been included.) Although menopause is a popular marker for the end of reproduction, age at menopause may covary with other measures of interest in any given study. Thus, menopausal women may be neither a random sample of women of completed fertility nor a random sample of any given age cohort.

In our analyses using the lifetime method, offspring survival was measured to 10 years and the sample was restricted to mothers who last gave birth at least 10 years prior to the interview. This was a conservative decision rule that allowed us to capture most of the mortality prior to age 20 years. In a survival analysis of 912 offspring, mortality was $19 \%$ by age 1 year, $43 \%$ by age 5 years, $47 \%$ by age 10 years, and $51 \%$ by age 20 years (Fig. 1). If we had chosen age 5 years, the sample size would have been $24 \%$ larger; thus, that would also have been a reasonable choice.

We defined our study population as the set of women, age 20 years and older, who were resident in the village of Sangui at any time during the decade from 1988-1998 (Strassmann and Gillespie, 2002). During the 10 years of this study only three women died between the time of their first live birth and menopause, and these three women were included in all analyses. The semiprospective nature of our study design reduced the problem of bias due to the omission of the reproductive histories of deceased adults. This bias is particularly problematic in cross-sectional studies of reproductive success (see Hill and Hurtado, 1996:83-101). Another way to approach this problem is to obtain reproductive histories of deceased adults from living parents or siblings, but, as noted by Hill and Hurtado (1983:91), such close kin may not exist. Hill and Hurtado provide a quantitative historical description of life history patterns among the Northern Ache from 1890-1993; thus, huge biases would have emerged if deceased individuals had been omitted. However, in studies of contemporary peoples with comparatively 


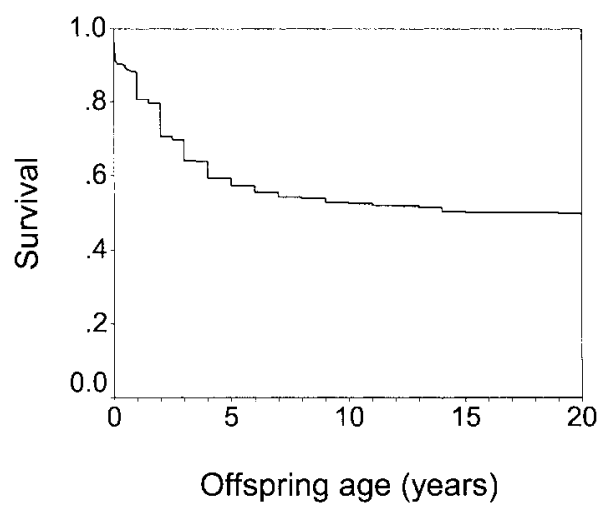

Fig. 1. Cumulative survival by age for a sample of 912 Dogon offspring.

low adult mortality (no warfare, ongoing epidemics, or high rates of homicide), interviews of third parties will sometimes be of less value. In particular, when the focus is on the covariates of RS, data from third parties may not be reliable. For example, Hill and Hurtado (1993:91) found that kin did not report accurately on each other's marriages or ages at puberty.

In sum, in implementing the lifetime method, long-term prospective data and third-party interviews provide two ways to minimize bias caused by the omission of the reproductive histories of the deceased. The lifetime method is otherwise free of inherent biases and is the closest to what might be called a "gold standard" for measuring reproductive success in the offspring generation. Nonetheless, this method often imposes a substantial limitation on sample size, which reduces its utility for identifying the covariates that predict RS. To avoid the problem of sample size reduction, researchers may turn to analyses of age-specific RS in samples that include women who may go on to have more births. Age-specific analyses may be retrospective or cross-sectional. They are not focused on older women, so they are less sensitive to longevity bias.

\section{AGE-SPECIFIC RETROSPECTIVE METHODS}

\section{Kaplan Meier-adjusted}

The Kaplan Meier-adjusted method is a retrospective technique for estimating age- specific RS (Strassmann and Gillespie, 2002). Compared with the lifetime method, it has the distinct advantage of increased sample size and correspondingly greater statistical power for detecting covariate effects. However, one constraint is that it requires data on age at death or censoring (the child's age at the parent's last interview). Another limitation is that it is best suited to highfertility populations. The first step when using this method is to obtain, for each woman, a Kaplan-Meier estimate (Kaplan and Meier, 1958) of the expected proportion of her offspring surviving to a given age, $\mathrm{x}$, such as 10 years. To estimate her age-specific $\mathrm{RS}$, each woman's estimate of the probability of offspring survivorship to age $\mathrm{x}$ is multiplied by the number of her livebirths at the time of her last interview.

The decision rule about which women to include in the sample can be liberal (yielding the largest sample size) or conservative. The most liberal rule will permit the inclusion of women whose oldest child was born at least $\mathrm{x}$ years prior to the date of interview. The most conservative rule, which permits only women whose youngest child was born at least $\mathrm{x}$ years prior to the date of interview, is indistinguishable from the lifetime method. Under either rule, $\mathrm{x}$ may assume a range of values, such as 5,10 , or 15 years.

The major limitation of the Kaplan Meieradjusted method is that it assumes that future survival probabilities at each age are the same as those already observed. This assumption may be unrealistic because a change in any number of factors could theoretically alter the probability of death, including wealth, kin support, marital status, maternal or paternal experience, and the number of offspring born, their sex, or their survival status. However, one can reduce the potential bias of these factors by increasing the required time since birth of the oldest offspring, which will mean longer follow-up for younger offspring and more complete survival data. One can also compare the results obtained with the most liberal decision rule against results obtained with the lifetime method.

When we made this comparison, the results of the two methods were in close agreement (Strassmann and Gillespie, 2002) (and see below). This concordance between the methods may imply that the above assumption was in fact met. Alternatively, by restricting the sample to women whose 
oldest offspring had reached age 10 years, we may have had sufficient follow-up on most younger offspring to accurately determine whether they had survived the first vulnerable years of life. Thus, our data may have captured most of the changes in mortality risk that occurred over time.

\section{Traditional}

The traditional age-specific retrospective method simply counts the number of offspring who survived to a given age, $\mathrm{x}$. This method differs from the lifetime method in that the sample is not restricted to women who last gave birth $\mathrm{x}$ years before the interview. Unlike the Kaplan Meier-adjusted method, children younger than age $\mathrm{x}$ are dropped from the analysis. Thus, compared with the Kaplan Meier-adjusted method, estimates of RS will be lower and some information is lost. However, the analysis is easier.

\section{CROSS-SECTIONAL METHODS}

Data are cross-sectional when the field worker only counts offspring who are alive at the time of the interview, but does not obtain data on age at death for deceased offspring, or age at censoring for living offspring. Cross-sectional data are appropriate when (a) subjects will freely discuss only those offspring who survived, or (b) when subjects deny knowledge of age at death for small children. For example, we found that among the Dogon, (a) applied to neither mothers nor fathers and (b) applied only to fathers. Cross-sectional methods may estimate lifetime or age-specific RS, depending on whether the women sampled are postreproductive.

\section{Lifetime}

In using the lifetime cross-sectional method, we restricted the sample to women who last gave birth 10 years prior to their last interview, making the sample the same as for the lifetime retrospective method. Compared with the retrospective method, the limitation of cross-sectional data is that offspring who reached age 10 years but who had died by the time of the inter- view are not counted. (Among these only those who died after sexual maturity bias the analysis because those who died beforehand would not themselves be reproductive.) To avoid undercounting reproductive offspring, deceased adults could be included in a person's RS if they were known to have reached age 20 years. This modification would be particularly helpful if adult mortality is high. The advantage of the crosssectional approach is that it makes it possible to estimate lifetime $\mathrm{RS}$ without requiring knowledge of offspring ages.

\section{Age-specific}

Another problem with cross-sectional data on offspring survival is that the RS of parents of younger offspring is exaggerated relative to the RS of parents of older offspring. This bias, which affects both the lifetime and age-specific approaches, arises because the cumulative risk of death always increases over time. The longer ago an offspring was born, the more likely it is that she was deceased by the time of the interview. The strengths of the cross-sectional methods are ease of data collection. The age-specific approach also permits larger sample size.

\section{IDENTIFYING COVARIATES}

To investigate covariates that may predict $\mathrm{RS}$ in a regression model, the age-specific RS estimates (Kaplan Meier-adjusted, traditional, and cross-sectional) must be adjusted for the overall pattern of increasing RS through the reproductive years. A plot of RS vs. age may help to establish whether a linear or nonlinear function is appropriate. With a large sample size, one might be able to detect the nonlinear effects caused by the inverse U-shaped relationship between fecundability and woman's age (Strassmann and Warner, 1998).

If age adjustment is used with retrospective data (the Kaplan Meier-adjusted and Traditional methods), then female RS will level off after the reproductive years. This plateau in RS can be handled by truncating the age of older women to approximately the average age at last birth plus the age to which survival is measured. For example, if the average age at last birth is 35 years and survival is measured to age 10 years, then all women age 45 years and older would be 
given the truncated age of 45 years. In most datasets, particularly those with smaller sample sizes, the data could then be fit by a linear model. When retrospective data are used for male RS, the relationship between male age and $\mathrm{RS}$ will depend on the mating system.

Models that use age adjustment with cross-sectional data will need to capture the increase in number of offspring through the reproductive years, followed by the decrease as adult offspring die. A quadratic function (putting age and age squared in the model) might adequately model this nonlinear relationship in both sexes (for males see, e.g., Strassmann, 2003).

Year of birth may be of interest as a predictor of RS independent of age. In particular, year of birth might be included in the model to capture cohort effects caused by, for example, a change in access to health care. However, unless the sample includes many postreproductive women interviewed over several decades, colinearity between age and year of birth may make it impossible to separate both effects. As with any continuous variable, nonlinear effects should be considered, such as a sudden increase in RS with the opening of a health clinic.

\section{EMPIRICAL COMPARISONS}

Figure 2 uses loess regression (Cleveland, 1993) to model the relationship between a woman's RS and her age, with RS calculated according to: the Kaplan Meier-adjusted method (Fig. 2a) the traditional method (Fig. 2b), and the cross-sectional method (Fig. 2c). These three graphs show considerable variability in $\mathrm{RS}$ at each age, reflecting differences in fertility and offspring mortality among women (Table 2). The two plots that use retrospective data show the expected leveling off in RS at age 45 or 50 years, whereas the cross-sectional plot shows the distinct downturn caused by the deaths of adult offspring prior to the mother's last interview.

Tables 3 and 4 compare the various methods for identifying predictors of RS among Dogon women. To facilitate comparison, all multiple regression models include the same covariates: mother's year of birth, marital rank (first wives and second through fourth wives vs. the reference category, sole wives), yabiru wives (who are in arranged
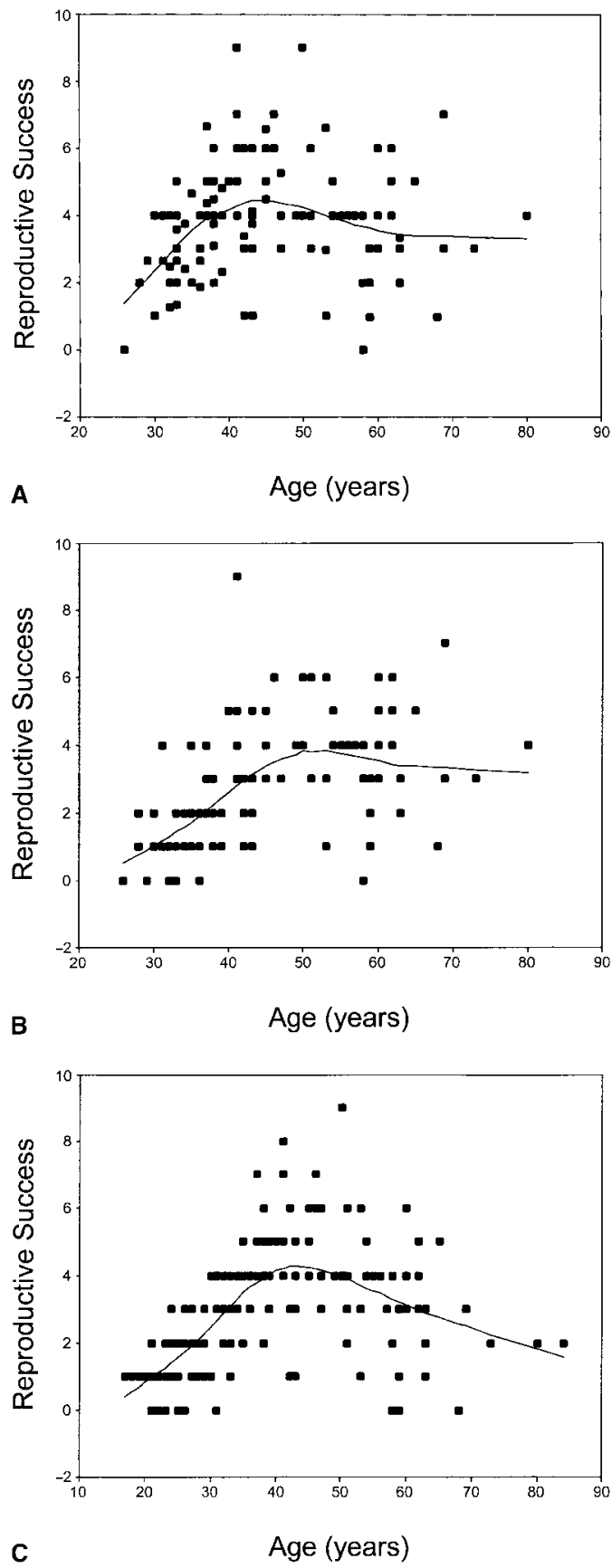

Fig. 2. Reproductive success (RS) plotted against woman's age with RS calculated according to: (A) the Kaplan Meier-adjusted method $(\mathrm{n}=117),(\mathbf{B})$ the traditional method $(\mathrm{n}=117)$, and $(\mathbf{C})$ the cross-sectional method $(\mathrm{n}=163)$. The loess regression (Cleveland, 1993) line is indicated. 
TABLE 2. Contribution of fertility ${ }^{a}$ and mortality $^{b}$ to reproductive success $^{c}$ ( $n=55$ women)

\begin{tabular}{lccccc}
\hline Reproductive success & N women & Mean fertility & SD fertility & Mean mortality & SD mortality \\
\hline 0 & 2 & 2.0 & 1.41 & 1.0 & 0.00 \\
1 & 6 & 7.0 & 3.22 & 0.7 & 0.36 \\
2 & 4 & 8.0 & 2.45 & 0.7 & 0.08 \\
3 & 16 & 7.5 & 2.66 & 0.6 & 0.27 \\
4 & 12 & 9.4 & 1.51 & 0.4 & 0.07 \\
5 & 7 & 8.0 & 1.41 & 0.3 & 0.21 \\
6 & 6 & 10.0 & 0.00 & 0.2 & 0.14 \\
$7-9$ & 2 & 8.1 & 2.56 & 0.5 & 0.26 \\
All women & 55 & & & & \\
\hline
\end{tabular}

From Strassmann and Gillespie (2002).

a Fertility is defined as the actual number of livebirths.

${ }^{\mathrm{b}}$ Mortality is defined as 1 minus the proportion of offspring surviving to age 10 years

${ }^{\mathrm{c}}$ Reproductive success is defined as the number of offspring who survived to age 10 years for women who last gave birth to a liveborn offspring at least 10 years prior to the interview.

marriages), yakezu wives (who are in nonarranged marriages), and levirate wives (who are married to the brother of a deceased husband). Levirate wives are the reference category because the other two wife categories were not significantly different when compared with each other.

We also include fertility and fertility squared as covariates, both of which were significant in most models. Predictors of RS may act through fertility; thus, one would not ordinarily include fertility along with the other covariates. However, among the Dogon the marital status variables influence child mortality (Strassmann, 1997, 2000) not fertility (Strassmann and Warner, 1998). In fact, most of the variation in RS among Dogon women is due to mortality (Table 2 ). Thus, for evaluating the marital status vari- ables as predictors of RS, it was helpful to adjust for fertility. This adjustment also takes care of the problem of incomplete reproductive histories for the models in Table 4, making adjustment for mother's age no longer necessary. We did, however, adjust for mother's year of birth, which was feasible because the correlation with fertility was sufficiently low $(r=0.46)$.

A second reason for including fertility as a covariate is that we are interested in the shape of the relationship between fertility and RS after controlling for the other covariates (Strassmann and Gillespie, 2002). In the lifetime retrospective model, which is the gold standard, and all other models except the one that estimates RS using the traditional method, the relationship between adjusted fertility and RS is clearly nonlinear.

TABLE 3. Predictors of reproductive success (the number of offspring who survived to age 10 years) by the two lifetime methods (retrospective and cross-sectional)

\begin{tabular}{|c|c|c|c|c|c|c|}
\hline \multirow[b]{2}{*}{ Predictor } & \multicolumn{3}{|c|}{$\begin{array}{l}\text { Lifetime retrospective method } \\
\qquad\left(\mathrm{n}=42^{\mathrm{f}}, \mathrm{r}^{2}=0.52\right)\end{array}$} & \multicolumn{3}{|c|}{$\begin{array}{l}\text { Lifetime cross-sectional method } \\
\qquad\left(\mathrm{n}=42^{\mathrm{f}}, \mathrm{r}^{2}=0.51\right)\end{array}$} \\
\hline & $\beta$ & $\mathrm{SE}$ & $P$ & $\beta$ & $\mathrm{SE}$ & $P$ \\
\hline Mother's year of birth & 0.05 & 0.026 & 0.071 & 0.06 & 0.026 & 0.019 \\
\hline 1st wife ${ }^{\mathrm{a}, \mathrm{c}}$ & -0.22 & 0.527 & 0.687 & -0.11 & 0.541 & 0.838 \\
\hline $2^{\text {nd }}, 3^{\text {rd }}, 4^{\text {th }}$ wife $\mathrm{e}^{\mathrm{a}, \mathrm{c}}$ & -0.58 & 0.583 & 0.325 & -0.661 & 0.598 & 0.227 \\
\hline Arranged wife $\mathrm{e}^{\mathrm{b}, \mathrm{c}}$ & -2.42 & 0.981 & 0.019 & -2.98 & 1.006 & 0.006 \\
\hline Nonarranged wife ${ }^{b, c}$ & -1.45 & 0.874 & 0.107 & -1.68 & 0.897 & 0.069 \\
\hline Fertility & 1.50 & 0.410 & 0.001 & 1.65 & 0.421 & 0.000 \\
\hline Fertility squared $^{\mathrm{d}}$ & -0.08 & 0.032 & 0.019 & -0.09 & 0.033 & 0.007 \\
\hline Constant & -93.51 & 50.480 & 0.073 & -126.32 & 51.771 & 0.020 \\
\hline
\end{tabular}

${ }^{\text {a }}$ Versus the omitted category, sole wife.

${ }^{\mathrm{b}}$ Versus the omitted category, levirate wife.

${ }^{c}$ Women who changed marital status during the study were assigned their marital status during two of three interviews. Thirteen widows were excluded from the analysis because they were not married at the time of the interviews and therefore did not have a prospectively observed marital status.

${ }^{\mathrm{d}}$ Corrected for the influence of mortality on fertility as described in Strassmann and Gillespie (2002). 
TABLE 4. Predictors of reproductive success (the number of offspring who survived to age 10 years) by the three age-specific methods (Kaplan Meier-adjusted, Traditional, and cross-sectional)

\begin{tabular}{|c|c|c|c|c|c|c|c|c|c|}
\hline \multirow[b]{2}{*}{ Predictor } & \multicolumn{3}{|c|}{$\begin{array}{l}\text { Kaplan Meier-adjusted method } \\
\qquad\left(\mathrm{n}=104, \mathrm{R}^{2}=0.46\right)\end{array}$} & \multicolumn{3}{|c|}{$\begin{array}{l}\text { Traditional method } \\
\left(\mathrm{n}=104, \mathrm{R}^{2}=0.48\right)\end{array}$} & \multicolumn{3}{|c|}{$\begin{array}{l}\text { Cross-sectional method } \\
\left(\mathrm{n}=150^{\mathrm{f}}, \mathrm{R}^{2}=0.66\right)\end{array}$} \\
\hline & $\beta$ & $\mathrm{SE}$ & $P$ & $\beta$ & SE & $P$ & $\beta$ & SE & $P$ \\
\hline Mother's year of birth & 0.05 & 0.012 & 0.000 & -0.03 & 0.012 & 0.025 & 0.05 & 0.009 & 0.000 \\
\hline 1st wife ${ }^{\mathrm{a}, \mathrm{c}}$ & -0.73 & 0.330 & 0.027 & -0.71 & 0.325 & 0.031 & -0.48 & 0.238 & 0.047 \\
\hline $2^{\text {nd }}, 3^{\text {rd }}, 4^{\text {th }}$ wife $e^{\mathrm{a}, \mathrm{c}}$ & -0.88 & 0.332 & 0.010 & -0.74 & 0.327 & 0.026 & -0.58 & 0.230 & 0.013 \\
\hline Arranged wife ${ }^{b, c}$ & -1.72 & 0.614 & 0.006 & -1.62 & 0.604 & 0.008 & -1.78 & 0.525 & 0.001 \\
\hline Nonarranged wife ${ }^{\mathrm{b}, \mathrm{c}}$ & -1.30 & 0.608 & 0.033 & -1.19 & 0.598 & 0.049 & -1.42 & 0.518 & 0.007 \\
\hline Fertility $^{\mathrm{d}}$ & 1.25 & 0.294 & 0.000 & 0.78 & 0.289 & 0.008 & 1.35 & 0.139 & 0.000 \\
\hline Fertility squared $^{\mathrm{d}}$ & -0.06 & 0.024 & 0.007 & -0.04 & 0.024 & 0.121 & -0.07 & 0.013 & 0.000 \\
\hline Constant & -89.27 & 23.26 & 0.000 & 53.41 & 22.90 & 0.022 & -96.37 & 18.565 & 0.000 \\
\hline
\end{tabular}

${ }^{\text {a }}$ Versus the omitted category, sole wife.

b Versus the omitted category, levirate wife.

${ }^{\mathrm{c}}$ Women who changed marital status during the study were assigned their marital status during two of three interviews. Thirteen widows were excluded from the analysis because they were not married at the time of the interviews and therefore did not have a prospectively observed marital status.

${ }^{\mathrm{d}}$ Corrected for the influence of mortality on fertility as described in Strassmann and Gillespie (2002).

e This model differs from the "age-adjustment model" in Strassmann and Gillespie (2002) in that mothers year of birth is substituted for age truncated to 50 years.

As seen by the negative and statistically significant coefficient for fertility squared (Tables 3 and 4), we observe diminishing returns to RS from higher fertility. This is an interesting finding in that two previous studies, both of forager populations, observed a linear increase in RS with fertility (Pennington and Harpending, 1988; Hill and Hurtado, 1996; but see Blurton Jones, 1986, 1997). The Dogon data, in contrast, provide clear evidence for a reproductive trade-off.

Table 3 compares the lifetime retrospective method against the lifetime cross-sectional method. Although the lifetime retrospective method is statistically unbiased, the coefficients for the two methods are similar as are most of the $P$-values. At $\alpha=0.05$, the significant covariates for the lifetime retrospective method are arranged (yabiru) wives, fertility, and fertility squared. Arranged wives had 2.4 fewer surviving offspring than levirate wives, on average. The significant covariates for the lifetime cross-sectional method are these same three plus mother's year of birth. For each decade later that a woman was born, she had 0.6 more surviving offspring on average. Given that it is often much more convenient and less invasive to simply inquire about living offspring, as opposed to ages at death for deceased offspring, these results suggest that the lifetime cross-sectional method, which is the simpler approach, may be adequate.
We note, however, that if we had not restricted the cross-sectional sample to women whose youngest child was born 10 years prior to her last interview, the results may not have been so similar. By restricting the sample in this way, we ensured that any child who died by age 10 would have already done so, making the two methods agree for children age 10 and under. The only difference between the methods is that the lifetime retrospective method counts children who died after age 10 (and prior to the interview) whereas the cross-sectional method does not.

Table 4 compares the three age-specific methods. The two retrospective methods have lower power and explain less of the variance $\left(\mathrm{n}=104, \mathrm{R}^{2}=0.46\right.$ or 0.48$)$ than the cross-sectional method $\left(\mathrm{n}=150, \mathrm{R}^{2}=0.68\right)$. However, for the most part, the coefficients and $P$-values are similar across the three methods. It is particularly interesting that the results of the cross-sectional method are similar to those of the more rigorous Kaplan Meier-adjusted method.

We suggest that this similarity reflects the Dogon demographic structure of high fertility and severe offspring mortality under age 5 years, but fairly strong survival thereafter. When a woman's offspring "alive at the time of the interview" are counted, most of the offspring who will die before age 10 will have already done so prior to the interview. Only the last one or two offspring has at most a 50\% chance of being erroneously 
classified as surviving. Although this does introduce a bias, the bias is relatively small. For these reasons the cross-sectional method is an extremely convenient and powerful method for detecting covariate effects in our study population. It would be expected to perform less well, however, in a population with either lower fertility or high mortality throughout childhood. In industrial populations with low offspring mortality throughout childhood, even age-specific fertility is probably an excellent measure of RS.

In both of the two retrospective methods, $\mathrm{RS}$ is based on the number of offspring who survived to age 10 years. Therefore, in both methods the sample is restricted to women ( $\mathrm{n}=104)$ who had at least one offspring who was born 10 years prior to the interview. These two methods have less power than the cross-sectional method $(\mathrm{n}=150)$, but the Kaplan Meier-adjusted method compensates for the loss of power by estimating the future survival of younger children. The traditional method simply discards all data on offspring who did not have 10 years of follow-up. The loss of data probably explains why, among the three age-specific methods, it was least sensitive for detecting covariate effects. In particular, the coefficient for fertility squared was smaller and the $P$-value was nonsignificant $(P=0.12)$. The unnecessary loss of data (and attendant risk of Type II errors) is a strong argument against the traditional method, although it is widely used. The traditional method is intended to avoid censoring bias, but the Kaplan Meieradjusted method provides a more fine-tuned means of correcting this problem.

\section{CONCLUSION}

We considered several alternative measures of reproductive success, defined for our retrospective analyses as the number of offspring who survived to age 10 years and for cross-sectional analyses as the number of children alive at the time of the interview. When an unbiased measure of lifetime RS is desired and little is known about the demographic structure of the population, then the lifetime retrospective method is preferable. However, it imposes a severe limitation on sample size that makes it insensitive for detecting covariate effects. The lifetime cross-sectional method gave very similar results and is unavoidable when data on offspring ages at death or censoring are unavailable. But it also lacks power.

Among the methods for estimating agespecific reproductive success, both the Kaplan Meier-adjusted method and the cross-sectional method were reasonable choices for the Dogon data. Neither discards data unnecessarily and both are sensitive for detecting covariate effects. Regression models that employed these two methods had very similar coefficients and $P$-values. These coefficients were also similar to those obtained using the lifetime retrospective method, but the standard errors for the coefficients were smaller. We conclude that in calculating or estimating $\mathrm{RS}$, human behavioral ecologists may choose among several options.

However, these options are contingent on the particularities of the field situation and the demographic structure of the study population. Choice of method had little impact on the results when using the Dogon data, but this might not be the case for other datasets. Further methodological comparisons using data from other study populations will be needed before our findings can be generalized to additional ethnographic contexts. In the absence of such studies, the cross-sectional methods should be used with caution.

\section{LITERATURE CITED}

Blurton-Jones N. 1986. Bushman birth spacing: a test for optimal interbirth intervals. Ethol Sociobiol 7: $91-105$.

Blurton-Jones N. 1997. Too good to be true? Is there really a trade-off between number and care of offspring in human reproduction? In: Betzig L, editor. Human nature: a critical reader. New York: Oxford University Press. p 83-86.

Cleveland WS. 1993. Visualizing data. Summit, NJ: Hobart Press.

Hill K, Hurtado M. 1996. Ache life history: the ecology and demography of a foraging people. New York: Aldine de Gruyter.

Kaplan EL, Meier P. 1958. Nonparametric estimation from incomplete observations. J Am Stat Assoc 53: 457-481.

Pennington R, Harpending H. 1988. Fitness and fertility among Kalahari !Kung. Am J Phys Anthropol 77: 303-319.

Strassmann BI. 1997. Polygyny as a risk factor for child mortality among the Dogon. Curr Anthropol 38: 688-695.

Strassmann BI. 2000. Polygyny, family structure, and child mortality: a prospective study among the Dogon of Mali. In: Cronk L, Chagnon N, Irons W, editors. Adaptation and human behavior: an anthropological perspective. New York: Aldine de Gruyter. p 49-67. 
Strassmann BI. 2003. Social monogamy in a human society: marriage and reproductive success among the Dogon. In: Reichard U, Boesch C, editors. Monogamy: mating strategies and partnerships in birds, humans and other mammals. Cambridge, UK: Cambridge University Press.
Strassmann BI, Gillespie B. 2002. Life-history theory, fertility and reproductive success in humans. Proc R Soc Lond B 269:553-562.

Strassmann BI, Warner J. 1998. Predictors of fecundability and conception waits among the Dogon of Mali. Am J Phys Anthropol 105:167-184. 